Received: 12.11 .2018

Revised: 12.12 .2018

Accepted: 14.12 .2018

DOI: $10.17804 / 2410-9908.2018 .6 .229-236$

\title{
STUDYING A FORCE ACTION ON A FLAT BOUNDLESS BARRIER FROM A GAS GENERATOR ACTIVATED UNDERWATER
}

\author{
I. I. Valov ${ }^{2 *}$ and Yu. P. Kabanov ${ }^{1}$ \\ ${ }^{I}$ Academician V. P. Makeyev State Rocket Centre JSC, Miass, Russian Federation \\ ${ }^{2}$ South Ural Scientific Centre, Ural Branch of RAS, Miass, Russian Federation \\ src@makeyev.ru; \\ 凶ofpat@mail.ru \\ *Corresponding author. E-mail: ofpat@mail.ru
}

The article aims to solve the problem of determination of a pressure field in liquid and a power load on elements of an object and test equipment at experimental tryout of underwater movement of objects. It presents a procedure of calculating the field of pressures on a flat barrier from the moment of underwater activation of a gas generator to touching of the barrier by a bubble. The barrier is replaced by a symmetrically arranged bubble of liquid, and the task is reduced to analyzing the movement of two spheres with varying radii and variable gas mass inside; the flow potential in this case has the form of the combination of Legendre polynomials. A law of a gas bubble evolution is derived, similar to the Rayleigh equation; the gas mass in the bubble is defined according to the Saint-Venant equation. Pressure distribution on the barrier is found using the CauchyLagrange integral. The developed methodology offers the possibility of evaluating loads on a moving object and a test bench at gas generator activation.

Keywords: gas generator, pressure distribution in liquid, experimental qualification.

\section{References}

1. Milne Thomson L.N. Theoretical Hydrodynamics, 4th ed., London, Macmillan, 1960.

2. Kostukov A.A. Vzaimodeistvie tel, dvizhushchikhsya v zhidkosti [Interaction of Bodies Moving in a Liquid]. Leningrad, Sudostroenie, 1972, 310 p. (In Russian).

3. Levkovsky Y.L. Struktura kavitatsionnykh techeniy [Structure of Cavitation Flows]. Sudostroenie Publishing House, Leningrad, 1978. (In Russian).

4. Volkov O.V., Petrov A.G. Motion of a variable-volume sphere in an ideal fluid near a plane surface. Fluid Dynamics, 1971, vol. 6, iss. 5, pp 808-817. DOI: 10.1007/BF01013864.

5. Voronin V.V., Kulikov V.N. The distribution of high-pressure gas jet in water at outflow from a submerged nozzle. In: Trudy TsAGI [TSAGI Proceedings], 1987, no. 2384, 16 p. (In Russian). 
Подана в журнал: 12.11.2018

УДК 532.5

DOI: $10.17804 / 2410-9908.2018 .6 .229-236$

\title{
ИССЛЕДОВАНИЕ СИЛОВОГО ВОЗДЕЙСТВИЯ НА ПЛОСКУЮ БЕЗГРАНИЧНУЮ ПРЕГРАДУ ПРИ ЗАПУСКЕ ПОД ВОДОЙ ГАЗОВОГО ГЕНЕРАТОРА
}

\author{
И. И. Валов ${ }^{2 *}$, Ю. П. Кабанов ${ }^{1}$ \\ ${ }^{1}$ Акиионерное общество «Государственный ракетный иентр имени академика В.П. Макеева» \\ 1, Тургоякское шоссе, г. Миасс, Российская Федерация \\ ${ }^{2}$ Федеральное государственное бюджетное научное учреждение «Южно-Уральский научный иентр», \\ 16, пр. Октября, г. Миасс, Российская Федераичи \\ ⿶sc@ makeyev.ru
ofpat@mail.ru \\ *Ответственный автор. Электронная почта: ofpat@mail.ru \\ Адрес для переписки: 16, пр. Октября, г. Миасс, 456317, Российская Федерация
}

Статья посвящена решению задачи определения поля давлений в жидкости и силовых нагрузок на элементы объекта и испытательного оборудования при экспериментальной отработке движения объектов под водой. Приводится методика расчетов по определению поля давлений на плоскую преграду от момента запуска под водой газового генератора до касания пузырем преграды. Преграда заменяется симметрично расположенным пузырем жидкости и задача сводится к исследованию движения двух сфер переменного радиуса с переменной массой газа в них, при этом потенциал течения представляется в виде комбинации полиномов Лежандра. Выводится закон развития газового пузыря, аналогичный уравнению Рэлея, масса газа в пузыре определяется в соответствии с уравнением Сен-Венана. Распределение давления на преграде находится с использованием интеграла Коши-Лагранжа. Построенная методика дает возможность оценить нагрузки на движущийся объект и экспериментальный стенд при запуске газового генератора.

Ключевые слова: газогенератор, поле давлений в жидкости, экспериментальная отработка.

\section{1. Введение}

При экспериментальной отработке подводного старта объектов, выходящих с подводных лодок, очень часто возникает необходимость определить поле давлений в жидкости и силовые нагрузки на элементы объекта и испытательного оборудования. Настоящая работа посвящена построению методики расчета силового воздействия струи на преграду при запуске газогенератора под водой от момента прорыва заглушки до подхода границы пузыря к поверхности преграды.

Поиск точного решения большинства практических задач гидродинамики, описываемых системами нелинейных дифференциальных уравнений (как правило, в форме задачи Коши или уравнений Навье-Стокса), сопряжен с большими трудностями, поэтому специалисты часто обращаются к численным методам решения. При этом для практика-экспериментатора, сталкивающегося с необходимостью в сжатые сроки спланировать и провести серию экспериментов, актуальна задача описания исследуемого процесса инженерной математической моделью, допускающей меньшую точность решения по сравнению с решением, полученным с помощью более точных сеточных методов, но позволяющей предсказать характер исследуемого процесса, диапазоны и периоды нарастания измеряемых величин, а также за короткое время просчитать несколько вариантов с различными условиями эксперимента. 
Инженерная модель развития газовой каверны, формируемой при выходе объекта из расположенной под водой стартовой установки, построена в монографии [6], а в работе [7] рассматриваются алгоритм решения задачи методом конечных элементов и приводятся результаты расчета. Построению и практической реализации методов решения задач механики сплошных сред, описывающих высокоинтенсивные процессы, сопровождающиеся значительным изменением одной из величин за малые промежутки времени, посвящены исследования $[8,9]$. Полученные результаты дают возможность существенно повысить точность решения и сократить необходимое для расчета время. Задача о движении в жидкости двух газовых сфер постоянного радиуса вдоль линии их центров рассматривалась в работах $[1,2]$, а движение пузырьков переменного радиуса с постоянной массой заключенных в них газов в работах $[3,4]$. В настоящей работе на основе модификации известных методов и математических моделей рассматривается движение двух сфер переменного радиуса с переменной массой газа и представлением потенциала течения в виде комбинации полиномов Лежандра. Задача ставится в форме Коши.

\section{2. Постановка задачи и методы решения}

Рассмотрим процесс взаимодействия струи с жидкостью от момента прорыва заглушки (рис. 1).

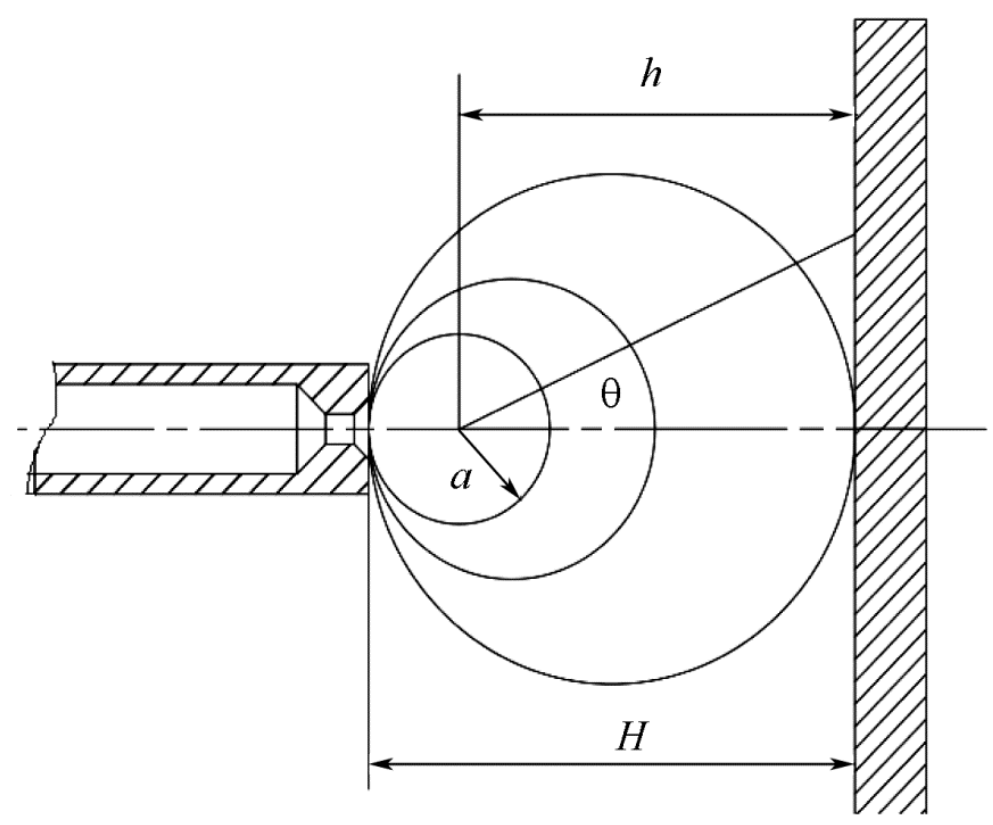

Рис. 1. Схема эксперимента

После прорыва заглушки у среза сопла в жидкости формируется газовый пузырь, который, развиваясь, воздействует на преграду, находящуюся на расстоянии $H$ от среза сопла. Задача решается при следующих допущениях:

- форма газового пузыря остается близкой к сферической, текущее расстояние от центра пузыря до преграды $h$ связано с радиусом пузыря $a$ как $h=H-a$. В силу кратковременности исследуемого процесса всплытием пузыря пренебрегаем;

- жидкость невязкая и несжимаемая, движение жидкости потенциальное. До начала запуска вода находится в состоянии покоя.

В этих предположениях определение воздействия струи на преграду можно свести к задаче о движении двух сфер переменного радиуса вдоль линии их центров (рис. 2), т. е. преграда заменяется симметрично расположенным пузырем в жидкости.

Valov I. I., Kabanov Yu. P. Studying a force action on a flat boundless barrier from a gas generator activated underwater // Diagnostics, Resource and Mechanics of materials and structures. - 2018. - Iss. 6. - P. 229-236. - DOI: 10.17804/2410- 


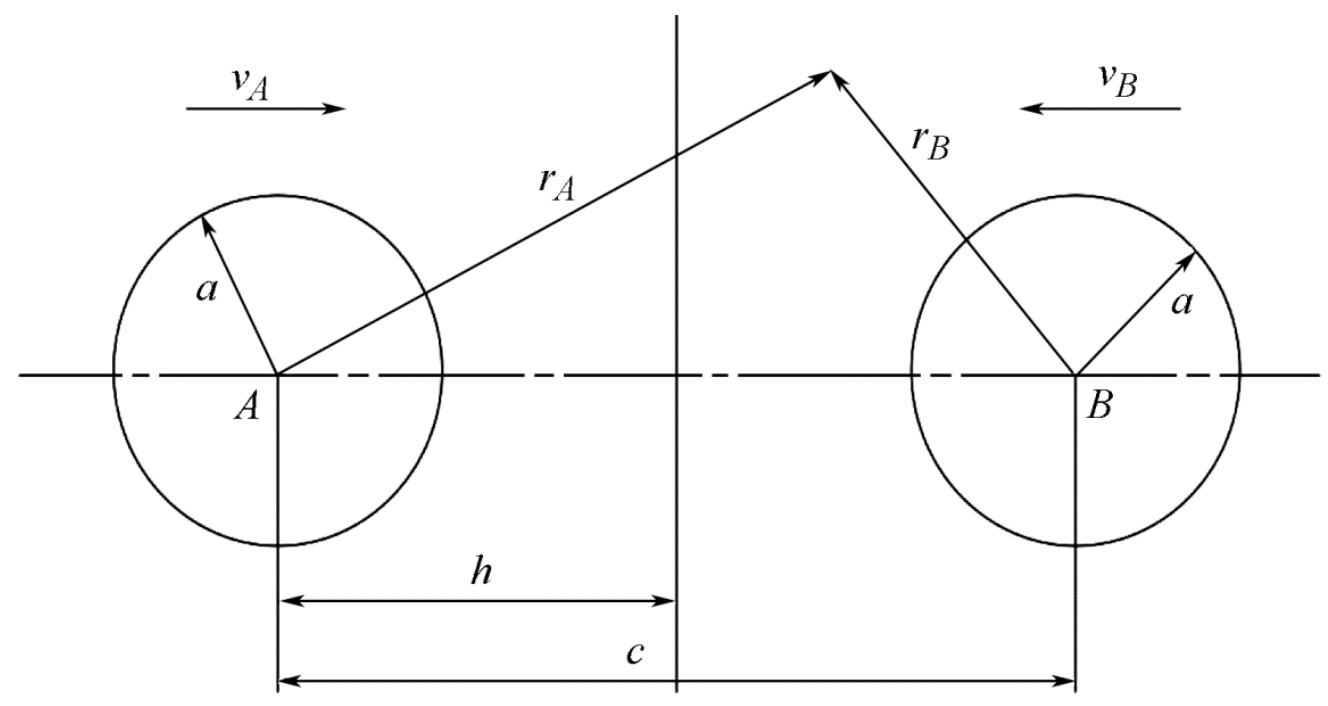

Рис. 2. Моделирование течения

Пусть сферы движутся навстречу друг другу с одинаковыми скоростями $v_{A}=v_{B}$. Расстояние между центрами шаров $c=2 h$. Потенциал скорости течения жидкости запишем в виде

$$
\Phi=\Phi_{A}+\Phi_{B}
$$

где каждая из функций удовлетворяет уравнению Лапласа и следующим граничным условиям:

- производные по коэффициентам функций $\Phi_{A}$ и $\Phi_{B}$ на бесконечности обращаются в ноль;

- на поверхности сфер

$$
\begin{gathered}
\left(\frac{\partial \Phi_{A}}{\partial r_{A}}\right)_{r_{A}=a}=\dot{a}+v_{A} \cos \theta_{A} \\
\left(\frac{\partial \Phi_{B}}{\partial r_{A}}\right)_{r_{A}=b}=0 ; \\
\left(\frac{\partial \Phi_{A}}{\partial r_{B}}\right)_{r_{B}=b}=0 ; \\
\left(\frac{\partial \Phi_{B}}{\partial r_{B}}\right)_{r_{B}=b}=\dot{b}+v_{B} \cos \theta_{B} .
\end{gathered}
$$

Таким образом, $\Phi_{A}$ представляет значение потенциала скорости для случая, когда сфера с центром в точке $A$ движется со скоростью $v_{A}$ по направлению к сфере с центром 
в точке $B$, в то время как сфера с центром в точке $B$ находится в покое. Аналогичный смысл имеет и функция $\Phi_{B}$. ния [2]:

Ограничимся рассмотрением расстояния вдоль преграды $r<c$, используя соотноше-

$$
\begin{gathered}
\frac{1}{r_{A}}=\frac{1}{c}\left[1+\frac{r_{B}}{c} P_{1}\left(\cos \theta_{B}\right)+\frac{r_{B}^{2}}{c^{2}} P_{2}\left(\cos \theta_{B}\right)+\ldots\right] ; \\
r_{A} \cos \theta_{A}=c-r_{B} \cos \theta_{B} .
\end{gathered}
$$

Представим потенциал течения в виде

$$
\begin{aligned}
& \Phi=a^{2} \dot{a}\left(\frac{1}{r_{A}}+\frac{1}{r_{B}}\right)+\frac{a^{3}}{2}\left[v_{A}+\frac{a^{2}}{c^{2}}\left(\dot{a}+\frac{a}{c} v_{A}\right)\right]+ \\
& {\left[\frac{P_{1}\left(\cos \theta_{A}\right)}{r_{A}^{2}}+\frac{P_{2}\left(\cos \theta_{B}\right)}{r_{B}^{2}}\right]+\frac{2 a^{7}}{3 c^{2}}\left(\dot{a}+\frac{3 a}{2 c} v_{A}\right)\left[\frac{P_{2}\left(\cos \theta_{A}\right)}{r_{A}^{3}}+\frac{P_{2}\left(\cos \theta_{B}\right)}{r_{B}^{3}}\right]+\ldots}
\end{aligned}
$$

$P_{n}(\cos \theta)-$ полиномы Лежандра,

$$
P_{1}(\cos \theta)=\cos \theta, P_{2}(\cos \theta)=\frac{3 \cos ^{2} \theta}{2}, \quad P_{n}(x)=\frac{1}{2^{n} n !} \frac{d^{n}}{d x^{n}}\left[\left(x^{2}-1\right)^{n}\right]
$$

Вблизи сферы $A$ потенциал потока можно представить в виде

$$
\begin{aligned}
& \Phi=a^{2} \dot{a}\left(\frac{1}{r_{A}}+\frac{1}{c}\left[1+\frac{r_{A}}{c} P_{1}\left(\cos \theta_{A}\right)+\frac{r_{A}^{2}}{c^{2}} P_{2}\left(\cos \theta_{A}\right)+\ldots\right]\right)+ \\
& +\frac{a^{3}}{2}\left[v_{A}+\frac{a^{2}}{c^{2}}\left(\dot{a}+\frac{a}{c} v_{A}\right)\right]\left\{\frac{P_{1}\left(\cos \theta_{A}\right)}{r_{a}^{2}}+\frac{1}{c^{2}}\left[1+\frac{2 r_{A}}{c} P_{1}\left(\cos \theta_{A}\right)+\frac{3 r_{A}^{3}}{c^{2}} P_{2}\left(\cos \theta_{A}\right)+\ldots\right]\right\}+\ldots .
\end{aligned}
$$

В условиях нашей задачи

$$
v_{A}=v_{B}=v, \quad v=-\dot{h}=\dot{a}, \quad h=H-a, \dot{a}=-\dot{h} .
$$

С учетом этого потенциал течения около газового пузыря

$$
\begin{aligned}
& \Phi=a^{2} \dot{a}\left\{\frac{1}{r}+\frac{1}{2 h}\left[1+\frac{r}{2 h} P_{1}\left(\cos \theta_{A}\right)+\frac{r^{2}}{4 h^{2}} P_{2}\left(\cos \theta_{A}\right)+\ldots\right]\right\}+ \\
& \frac{a^{3}}{2}\left[\dot{a}+\frac{a^{2}}{4 h^{2}}\left(\dot{a}+\frac{a}{2 h} \dot{a}\right)\right]\left\{\frac{P_{1}\left(\cos \theta_{A}\right)}{r^{2}}+\frac{1}{4 h^{2}}\left[1+\frac{r}{h} P_{1}\left(\cos \theta_{A}\right)+\frac{3}{4} \frac{r^{2}}{h^{2}} P_{2}\left(\cos \theta_{A}\right)+\ldots\right]\right\}+\ldots .
\end{aligned}
$$

Используя интеграл Коши-Лагранжа, можно определить давление около газового пузыря

Valov I. I., Kabanov Yu. P. Studying a force action on a flat boundless barrier from a gas generator activated underwater // Diagnostics, Resource and Mechanics of materials and structures. - 2018. - Iss. 6. - P. 229-236. - DOI: 10.17804/24109908.2018.6.229-236. 


$$
\frac{P-P_{0}}{\rho}=\frac{\partial \Phi}{\partial t}-\frac{1}{2}\left[\left(\frac{\partial \Phi}{\partial r}\right)^{2}+\left(\frac{1}{r} \frac{\partial \Phi}{\partial \theta}\right)^{2}\right] .
$$

В уравнении (1) члены, не зависящие от угла $\theta$, определяют общее расширение газового пузыря:

$$
\frac{P_{n y 3}-P_{0}}{\rho}=a \ddot{a}\left(1-\frac{a^{2}}{4 h^{2}}+\frac{1}{12} \frac{a^{3}}{h^{3}}\right)+\dot{a}^{2}\left(\frac{3}{2}+\frac{a}{h}-\frac{19}{24} \frac{a^{3}}{h^{3}}+\frac{1}{8} \frac{a^{2}}{h^{2}}\right)
$$

с точностью до членов $\frac{a^{4}}{h^{4}}$ порядка малости.

Используя уравнение состояния идеального газа, получим дифференциальное уравнение второго порядка относительно $a$ :

$$
a \ddot{a}\left(1-\frac{a^{2}}{h^{2}}+\frac{1}{12} \frac{a^{3}}{h^{3}}\right)+\dot{a}^{2}\left(\frac{3}{2}+\frac{a}{h}+\frac{1}{8} \frac{a^{2}}{h^{2}}-\frac{19}{24} \frac{a^{3}}{h^{3}}\right)=\frac{G R T}{\frac{4}{3} \pi \rho a^{3}}-\frac{P_{0}}{\rho}
$$

с начальными условиями при $t=0$

$$
\begin{aligned}
& a=a_{0} \\
& \dot{a}=0 \\
& \ddot{a}=\frac{\left(P_{1}-P_{0}\right)}{\rho a_{0}\left(1-\frac{a_{0}^{2}}{4 h^{2}}+\frac{1}{12} \frac{a^{3}}{h^{3}}\right)} \approx \frac{P_{1}-P_{0}}{\rho a_{0}} .
\end{aligned}
$$

Здесь $G$ - суммарный вес газов в пузыре

$$
G(t)=\int_{0}^{t} \dot{G} d t
$$

Температура газа в пузыре $T$ изменяется в соответствии с законом [5]:

$$
\frac{d T}{d t}=\frac{1}{G}\left[\dot{G}\left(k * T_{K C}-T\right)-\frac{P}{C_{V}} 2 \pi a \dot{a}\right]
$$

Разрешая уравнение (3) с учетом (2) и (4), получим зависимость $a, \dot{a}, \ddot{a}$ как функцию времени $t$, при этом расход газов из газогенератора определяется по формулам Сен-Венана с учетом противодавления в газовом пузыре:

$$
\dot{G}= \begin{cases}\mu C D, & \text { если } \frac{P_{0}}{P_{K C}}>\left(\frac{2}{k+1}\right)^{\frac{k}{k-1}} \\ \mu C, & \text { если } \frac{P_{0}}{P_{K C}} \leq\left(\frac{2}{k+1}\right)^{\frac{k}{k-1}}\end{cases}
$$

где

Valov I. I., Kabanov Yu. P. Studying a force action on a flat boundless barrier from a gas generator activated underwater // Diagnostics, Resource and Mechanics of materials and structures. - 2018. - Iss. 6. - P. 229-236. - DOI: 10.17804/24109908.2018.6.229-236. 


$$
\begin{gathered}
C=S P_{K C}\left(\frac{2}{k+1}\right)^{\frac{k+1}{2(k-1)}} \sqrt{\frac{k}{R T_{K C}}} \\
D=\sqrt{\frac{2}{k-1}\left(\frac{k+1}{2}\right)^{\frac{k+1}{k-1}}\left(\frac{P_{0}}{P_{K C}}\right)^{4 k}\left[1-\left(\frac{P_{0}}{P_{K C}}\right)^{\frac{k-1}{k}}\right]}
\end{gathered}
$$

$S$ - площадь минимального (критического) сечения сопла; $\mu$ - коэффициент расхода; $P_{K C}, T_{K C}-$ давление и температура в камере сгорания газового генератора соответственно; $k$ - показатель адиабаты продуктов сгорания.

Учитывая связь между точками в системе координат, связанных с пузырем $r_{A} \cos \theta_{A}=c-r_{B} \cos \theta_{B}$, а также соотношения

$$
v=-\dot{h}=\dot{a} ; \dot{\theta}=\frac{v \sin \theta}{r} ; c=2 h
$$

и используя уравнение Коши-Лагранжа, получим выражение для распределения давления на преграде $P_{n}$

$$
\frac{P_{n}-P_{0}}{\rho}=\frac{\partial \Phi}{\partial t}-\frac{1}{2}\left[\left(\frac{\partial \Phi}{\partial r}\right)^{2}+\left(\frac{1}{r} \frac{\partial \Phi}{\partial \theta}\right)^{2}\right],
$$

где

$$
\begin{gathered}
\frac{\partial \Phi}{\partial t}=\frac{a^{2} \ddot{a}}{(H-a)} \cos \theta\left[2+\frac{a \cos ^{2} \theta}{(H-a)}\left(1-\frac{a^{2}}{4(H-a)^{2}}\right)\right]+\frac{4 a \dot{a}^{2} \cos \theta}{(H-a)}+\frac{5 a^{2} \dot{a} \cos ^{3} \theta}{(H-a)^{2}}\left(1+\frac{a^{2}}{4(H-a)^{2}}\right)+ \\
+\frac{a^{3} \dot{a}^{2} \cos ^{3} \theta}{(H-a)^{3}}\left(2 \cos ^{2} \theta-\sin ^{2} \theta\right)\left(1+\frac{a^{2}}{4(H-a)^{2}}\right) \\
\frac{\partial \Phi}{\partial r}=\frac{a^{2} \dot{a} \cos \theta}{(H-a)^{2}}(1-\cos \theta)\left[1-\frac{a}{(H-a)}+\frac{a^{3}}{4(H-a)^{3}}\right] \\
\frac{1}{r} \frac{\partial \Phi}{\partial \theta}=\frac{a^{2} \dot{a} \sin \theta}{(H-a)^{2}}\left[1-\frac{a \cos \theta}{(H-a)}+\frac{a^{3} \cos \theta}{(H-a)^{3}}+\frac{a^{4} \cos \theta}{(H-a)^{3}}\right]
\end{gathered}
$$

Valov I. I., Kabanov Yu. P. Studying a force action on a flat boundless barrier from a gas generator activated underwater // Diagnostics, Resource and Mechanics of materials and structures. - 2018. - Iss. 6. - P. 229-236. - DOI: 10.17804/24109908.2018.6.229-236. 
Окончательное выражение для избыточного давления на преграде запишется как

$$
\begin{aligned}
& \frac{\Delta P_{n}}{\rho}=\frac{a^{2} \ddot{a}}{(H-a)} \cos \theta\left[2+\frac{a \cos ^{2} \theta}{(H-a)}+\frac{a^{3} \cos ^{2} \theta}{4(H-a)^{3}}\right]+ \\
& +\frac{a \dot{a}^{2} \cos \theta}{(H-a)}\left\{\left[4+\frac{5 a \cos ^{2} \theta}{(H-a)}+\frac{5}{4} \frac{a^{3} \cos ^{2} \theta}{(H-a)^{3}}\right]+\frac{a^{2} \cos ^{2} \theta}{(H-a)^{2}}\left(2 \cos ^{2} \theta-\sin ^{2} \theta\right)\left(1+\frac{a^{2}}{4(H-a)^{2}}\right)\right\}- \\
& -\frac{a^{4} \dot{a}^{2} H^{2}}{(H-a)^{6}}\left\{(1-\cos \theta)^{2}+\sin ^{2} \theta\left[1-\frac{a}{H}(1-\cos \theta)\right]^{2}\right\}
\end{aligned}
$$

Подставляя в выражение (6) значение радиуса, скорости и ускорения изменения радиуса пузыря, можно найти значение давления на преграде в любой точке, находящейся на расстоянии $r=(H-a) \operatorname{tg} \theta$ от центра преграды. При этом следует учесть, что полученные формулы справедливы при $r<2 h$, что на момент касания пузырем преграды составляет $r<\frac{3}{2} H$

\section{3. Заключение}

Приведенная методика расчета дает возможность определить поле давлений при запуске под водой газового генератора и оценить влияние ряда конструктивных параметров на возникающие при этом нагрузки на модель объекта и экспериментальный стенд.

\section{Литература}

1. Милн-Томпсон Л. М. Теоретическая гидродинамика / пер. с английского. - М. : Мир, 1964. $-660 \mathrm{c}$.

2. Костюков А. А. Взаимодействие тел, движущихся в жидкости. - Л. : Судостроение, 1972. $-310 \mathrm{c}$.

3. Левковский Ю. Л. Структура кавитационных течений. - Л. : Судостроение, 1978. $224 \mathrm{c}$.

4. Voinov O. V., Petrov A. G. Motion of a variable-volume sphere in an ideal fluid near a plane surface // Fluid Dynamics. - 1971. - Vol. 6, iss. 5. - P. 808-817. - DOI: 10.1007/BF01013864.

5. Воронин В. В., Куликов В.Н. Распространение высоконапорной газовой струи в воде при истечении из затопленного сопла // Труды ЦАГИ, вып. 2384. - М. : 1987. 